Imperial/TP/97-98/33

hep-th/9803184

To appear Phys.Lett.B.

23rd March 1998, final corrections 8th April 1999

Present version 9th April 1999

\title{
Regularization schemes and the multiplicative anomaly
}

\author{
T.S. Evans* \\ Theoretical Physics, Blackett Laboratory, Imperial College, \\ Prince Consort Road, London, SW7 2BZ, U.K.
}

\begin{abstract}
Elizalde, Vanzo, and Zerbini have shown that the effective action of two free Euclidean scalar fields in flat space contains a 'multiplicative anomaly' when $\zeta$ function regularization is used. This is related to the Wodzicki residue. I show that there is no anomaly when using a wide range of other regularization schemes and that the anomaly can be removed by an unusual choice of renormalization scales. I define new types of anomalies and show that they have similar properties. Thus multiplicative anomalies encode no novel physics. They merely illustrate some dangerous aspects of $\zeta$-function and Schwinger proper time regularization schemes.
\end{abstract}

In QFT (quantum field theory) one often encounters terms of the form $\ln \operatorname{det}\left(\Delta^{-1}\right)$ where $\Delta$ is the propagator for some field. This arises for example in one loop approximations to effective actions. However, in QFT this is a determinant of an infinite dimensional matrix, since there are an infinite number of physical modes. It is therefore of great interest to all practitioners of QFT that the "multiplicative anomaly" $a(A, B)[1,2]$, where

$$
a(A, B):=\ln \operatorname{det}(A B)-\ln \operatorname{det}(A)-\ln \operatorname{det}(B),
$$

need not be zero if $A, B$ are infinite matrices. The term anomaly refers to the failure of a standard algebraic expression rather than its traditional use in QFT in relation to symmetries. As usual in QFT the individual terms in (1) are naively infinite, so one must regulate the ultra-violet before any serious discussion. I will indicate that some regularization scheme, labelled $R$, has been used by adding a subscript $R$, e.g. $a_{R},[\ln \operatorname{det}(A)]_{R}$.

So far the anomaly has only been considered using $\zeta$-function regularization [2]. In particular, if $A$ and $B$ are elliptic operators and $\zeta$-function regularization is used then

*email: T.Evans@ic.ac.uk, WWW: http://euclid.tp.ph.ic.ac.uk/ time 
the anomaly $a_{\zeta}$ is not generally zero. This $a_{\zeta}$ is related to the Wodzicki residue $[3,1]$ which has been used in the non-commutative geometric approach to particle physics [4]. Recently Elizalde, Vanzo and Zerbini [2] showed that the multiplicative anomaly in $\zeta$ function regularization produces surprising results in the simplest of QFT problems. For instance, for two real free relativistic scalar fields of mass $m_{1}, m_{2}$ respectively, in flat $D$ dimensional Euclidean space-time of space-time volume $\mathcal{V}_{D}, D$ an even positive integer, [2] gives the anomaly as

$$
a_{\zeta}\left(\Delta_{1}^{-1}, \Delta_{2}^{-1}\right)=\mathcal{V}_{D} \frac{(-1)^{D / 2}\left(m_{1}^{2}-m_{2}^{2}\right)^{D / 2}}{D C(D)} \cdot(\Psi(1)-\Psi(D / 2)),
$$

where $\Delta_{j}^{-1}=-\partial^{2}+m_{j}^{2}$, and $C(d)=(4 \pi)^{d / 2} \cdot \Gamma(d / 2)$.

At first sight this result is surprising - how can two free scalar fields mix in this way? In fact in QFT such free fields could be mixed by choosing a vacuum with a condensate mixing the two fields and perhaps the calculation is revealing this possibility. For instance, neutrino mixing and kaon oscillations can be modelled using a simple quadratic Lagrangian with no mixing/interaction terms if one chooses a non-standard vacuum [5, 6].

In this letter, I ask why such anomalies have not been noted in standard QFT texts. To do this I extend the definition of the anomaly of [2] from one defined in terms of $\zeta$ function regularisation to a wide variety of popular regularization schemes. I also define two new types of anomaly to illustrate that there are a large number of such multiplicative anomalies. One conclusion is that the existence of the multiplicative anomalies depends on the type of regularization scheme used. The analysis here suggests a general principle, namely that only mass-dependent regularization schemes can produce anomalies. Thus simple sharp momentum cutoffs and dimensional regularization usually have no multiplicative anomalies. On the other hand, $\zeta$-function regularization and all the other Schwinger proper time regularizations [11] change the propagators so that they are mass-dependent schemes which leads to anomalies. Since physics must be independent of the regularization scheme, (though this will only be approximately true in a general approximation scheme [9] and [19]) and anomalies are zero in some schemes, this shows that multiplicative anomalies contain no new physics. They are merely a technical complication of some regularization schemes. At the very least they are equivalent to a finite shift in counter terms and I will indeed show that they are equivalent to a shift in the renormalization scales. Thus this analysis suggests that $\zeta$-function regularization is a difficult scheme to use in flat-space time where changes to the analytic structure of the propagators are involved e.g. a self-energy shift. Yet $\zeta$-function regularization has been of great use in curved space-time, so the role of the multiplicative anomaly in such calculations needs to be examined.

Consider the example of two free scalar fields. One formal definition of the partition function, $Z$, is

$$
\begin{aligned}
Z & =\int D \phi_{1} D \phi_{2} \exp \left\{-\int d^{D} x\left(\phi_{1} \Delta_{1}^{-1} \phi_{1}+\phi_{2} \Delta_{2}^{-1} \phi_{2}\right)\right\}=e^{-W_{1}} \cdot e^{-W_{2}}, \\
W_{j} & =\frac{1}{2} \ln \operatorname{det}\left(\Delta_{j}^{-1} / \mu_{j}^{2}\right),
\end{aligned}
$$

where I have anticipated renomalisation by including renormalization scales $\mu_{j}$. The partition function may also be written as in terms of a vector of fields $(\vec{\Phi})_{j}=\phi_{j}$ with 
mass matrix $M_{i j}^{2}=m_{i}^{2} \delta_{i j}(i, j=1,2)$,

$$
\begin{aligned}
Z & =\int D \vec{\Phi} \exp \left\{-\int d^{D} x \Phi_{i}\left(-\partial^{2} \delta_{i j}+M_{i j}^{2}\right) \Phi_{j}\right\} \\
& =\left[\operatorname{DET}\left\{\left(-\partial^{2} \delta_{i j}+M_{i j}^{2}\right) / \mu_{12}^{2}\right\}\right]^{-1 / 2}=\exp \left(-W_{12}\right) \\
& =\left[\operatorname{det}\left\{\left(\left(\partial^{2}\right)^{2}-\partial^{2}\left(m_{1}^{2}+m_{2}^{2}\right)+m_{1}^{2} m_{2}^{2}\right) / \mu_{12}^{4}\right\}\right]^{-1 / 2} \\
W_{12} & =\frac{1}{2} \ln \operatorname{det}\left(\left\{\left(\partial^{2}\right)^{2}-\partial^{2}\left(m_{1}^{2}+m_{2}^{2}\right)+m_{1}^{2} m_{2}^{2}\right\} / \mu_{12}^{4}\right)
\end{aligned}
$$

Note that the direct calculation of the gaussian in a product space of space-time and fields ( $x$ and $i$ indices respectively) produces the result (5), with the determinant over this product space denoted by DET. However, moving to a determinant just over spacetime indices, denoted by det, is not usually trivial as pointed out by Dowker [7]. Here though, the matrix is diagonal in the field indices and in this special case Dowker's general formula confirms that (7) follows from (5).

Finally one can formally factorize the operator and then the determinant in $W_{12}$ of $(7)$ to reproduce (3). The anomaly $a\left(\Delta_{1}^{-1}, \Delta_{2}^{-1}\right)=2 W_{12}-2 W_{1}-2 W_{2}$ expresses the failure of this factorisation. However, first one must regulate these UV infinite expressions.

The standard regularization scheme of modern particle physics is dimensional regularization $[8,9]$ which gives

$$
\begin{aligned}
2\left[W_{j}\right]_{\epsilon} & =\mathcal{V}_{D} \int \frac{d^{d_{j}} k}{(2 \pi)^{d_{j}}} \ln \left(\frac{k^{2}+m_{j}^{2}}{\mu_{j}^{2}}\right) \\
2\left[W_{12}\right]_{\epsilon} & =\mathcal{V}_{D} \int \frac{d^{d_{12}} k}{(2 \pi)^{d_{12}}} \ln \left(\frac{\left(k^{2}+m_{1}^{2}\right)\left(k^{2}+m_{2}^{2}\right)}{\mu_{12}^{4}}\right)
\end{aligned}
$$

where $d_{x}=D-2 \epsilon_{x}, D \in \mathbb{Z}^{+}, \forall x \in\{1,2,12\}$, and we use an $\epsilon$ subscript to label quantities calculated in dimensional regularization. For the simple problems considered here, dimensional regularization is merely changing the loop integration to

$$
\left[\int \frac{d^{d} k}{(2 \pi)^{d}}\right]_{\epsilon}=\frac{1}{C(d)} \int_{0}^{\infty} d K K^{D / 2-1} \frac{K}{\mu^{2}}-\epsilon
$$

where $K=k^{2}$. For later use, note that it is clearly a mass independent regularization scheme provided the renormalization scale, $\mu$, remains an independent parameter. We quickly see that the anomaly in dimensional regularization is

$$
a_{\epsilon}\left(\Delta_{1}^{-1}, \Delta_{2}^{-1}\right)=2\left[W_{12}\right]_{\epsilon}-2\left[W_{1}\right]_{\epsilon}-2\left[W_{2}\right]_{\epsilon}=0
$$

provided we choose $\epsilon_{12}=\epsilon_{1}=\epsilon_{2}$ and $\mu_{12}=\mu_{1}=\mu_{2}$. This result follows because once regularized we can manipulate the integrals freely and it is legitimate to combine the logarithms of the integrands in (8) and (9) into a single logarithm under one integration. In a similar way we can see that any regularization scheme which works by altering the range or measure of loop momenta integrations (denoted by LR) using a single renormalization scale $\mu$ has no anomaly, $a_{\mathrm{LR}}=0$, as all the integrals involved are well behaved.

$$
\begin{aligned}
& a_{\mathrm{LR}}\left(\Delta_{1}^{-1}, \Delta_{2}^{-1}\right):=2\left[W_{12}\right]_{\mathrm{LR}}-2\left[W_{1}\right]_{\mathrm{LR}}-2\left[W_{2}\right]_{\mathrm{LR}} \\
& \quad=\mathcal{V}_{D}\left[\int \frac{d^{D} k}{(2 \pi)^{D}}\right]_{\mathrm{LR}}\left\{\ln \left(\frac{\left(k^{2}+m_{1}^{2}\right)\left(k^{2}+m_{2}^{2}\right)}{\mu^{4}}\right)-\ln \left(\frac{k^{2}+m_{1}^{2}}{\mu^{2}}\right)-\ln \left(\frac{k^{2}+m_{2}^{2}}{\mu^{2}}\right)\right\} \\
& =0
\end{aligned}
$$


For the case of a sharp momentum cutoff, there can be no doubt that the above analysis is correct. Since dimensional regularization has some technical complications, e.g. see [22], I will give a more detailed analysis below.

Before that, consider a scheme where $\ln (A) \rightarrow\left(-s A^{s}\right)^{-1}[10]$ which I will call $s$ regularization. This is one of the family of Schwinger proper time regularizations [11]. Thus one defines

$$
\begin{aligned}
2\left[W_{j}\right]_{s} & =-\mathcal{V}_{D} \frac{1}{s_{j}} \int \frac{d^{D} k}{(2 \pi)^{D}}\left(\frac{\mu_{j}^{2}}{\left(k^{2}+m_{j}^{2}\right)}\right)^{s_{j}}, \\
2\left[W_{12}\right]_{s} & =-\mathcal{V}_{D} \frac{2}{s_{12}} \int \frac{d^{D} k}{(2 \pi)^{D}}\left(\frac{\mu_{12}^{4}}{\left(k^{2}+m_{1}^{2}\right)\left(k^{2}+m_{2}^{2}\right)}\right)^{s_{12} / 2} .
\end{aligned}
$$

As a series about $s_{x}=0$, these expressions have a simple pole, a constant term and higher order corrections just like the small $\epsilon$ dimensional regularized results. Note that the physical masses are clearly an integral part of the regulatory mechanism making this a mass-dependent scheme. The anomaly in the $s$-regularization scheme is then found to be $a_{s}\left(\Delta_{1}^{-1}, \Delta_{2}^{-1}\right)=2\left[W_{12}\right]_{s}-2\left[W_{1}\right]_{s}-2\left[W_{2}\right]_{s} \neq 0$. Again the integrals are all finite for non-zero but small $s$ so the integrands can be combined. However, the result is obviously not zero because $2(A B)^{-s / 2} \neq(A)^{-s}+(B)^{-s}$. The whole family of Schwinger propertime regularizations [11] of which $s$-regularization is the simplest example, give the same result.

To make contact with the $\zeta$-function regularization scheme $[10,11,12]$ used in [2] one applies

$$
\zeta(s \mid A)=\frac{1}{\Gamma(s)} \int_{0}^{\infty} d t t^{s-1} \operatorname{Tr}\left\{e^{-t A}\right\}
$$

to (14) and (15) to prove that

$$
2\left[W_{A}\right]_{s}=-\frac{1}{s} \zeta(s \mid A)=-\frac{1}{s} \zeta(0 \mid A)+2\left[W_{A}\right]_{\zeta}+O(s), \quad 2\left[W_{A}\right]_{\zeta}:=\left.\frac{d}{d s} \zeta(s \mid A)\right|_{s=0}
$$

where $\exp \left\{-W_{A}\right\}=\int D \phi \exp \{-\phi A \phi\}$. Thus the $\zeta$-function method in this context is equivalent to throwing away the diverging term of the $s$-regularized expressions. So $\zeta$ function 'regularisation' is to Schwinger regularisation schemes what the MS (minimal subtraction) renormalization scheme is to dimensional regularisation. Since the divergent parts of the $s$-regularization cancel in the anomaly $a_{s}$, it follows that $a_{s}\left(\Delta_{1}^{-1}, \Delta_{2}^{-1}\right)=$ $a_{\zeta}\left(\Delta_{1}^{-1}, \Delta_{2}^{-1}\right) \neq 0$, in agreement with $(2)$ of $[2]$.

To allay any suspicions that there is some slight of hand hiding in the manipulation of infinite quantities, I will present a generalisation of a calculation in [2]. Since dimensional regularization alters the momentum integration, while $s$ - and $\zeta$-regularizations involve changes to the propagators, I can combine these two techniques. Thus I consider the anomaly using a mixed "se-regularization", $a_{s \epsilon}\left(\Delta_{1}^{-1}, \Delta_{2}^{-1}\right)=2\left[W_{12}\right]_{s \epsilon}-2\left[W_{1}\right]_{s \epsilon}-2\left[W_{2}\right]_{s \epsilon}$, where

$$
\begin{aligned}
2\left[W_{j}\right]_{s \epsilon} & =-\mathcal{V}_{D} \frac{1}{s_{j}} \mu_{j}^{2 s_{j}+2 \epsilon_{j}} \int \frac{d^{d_{j}} k}{(2 \pi)^{d_{j}}}\left(k^{2}+m_{j}^{2}\right)^{-s_{j}} \\
2\left[W_{12}\right]_{s \epsilon} & =-\mathcal{V}_{D} \frac{2}{s_{12}} \mu_{12}^{2 s_{12}+2 \epsilon_{12}} \int \frac{d^{d_{12}} k}{(2 \pi)^{d_{12}}}\left(\left(k^{2}+m_{1}^{2}\right)\left(k^{2}+m_{2}^{2}\right)\right)^{-s_{12} / 2} .
\end{aligned}
$$


To calculate the anomaly I use the same approach as [2] and assume that it is a function only of the mass difference, so I must set $\mu_{1}=\mu_{2}$. Thus by taking the $m_{2}^{2}=0$ limit and using standard integrals, [18],

$$
\int_{0}^{\infty} d x x^{\mu-1}(1+x)^{-\nu}=\frac{\Gamma(\mu) \Gamma(\nu-\mu)}{\Gamma(\nu)}
$$

I find the following result for $D=4$ dimensions

$$
a_{s \epsilon}\left(\Delta_{1}^{-1}, \Delta_{2}^{-1}\right)=\mathcal{V}_{4} \frac{\left(m_{1}^{2}-m_{2}^{2}\right)^{2}}{4 C(4)}\left(\frac{1}{1+r}(\Psi(2)-\Psi(1))+\ln \left(\frac{\mu_{12}}{\mu_{1}}\right)\right)
$$

where $r:=\lim _{s, \epsilon \rightarrow 0}(\epsilon / s)$. I also choose $\epsilon=\epsilon_{x}, s=s_{x} \forall x \in\{1,2,12\}$ as this is essential if the UV divergences are to cancel.

Let us first set the renomalisation scales $\mu_{x}$ equal, as in [2] and most other work. The pure $s$ - and $\zeta$-regulated answers come from putting $\epsilon=0(r=0)$. Thus we are not surprised that setting $\epsilon=0, \mu_{12}=\mu_{1}$ in (18) confirms the result (2) of [2] (apart from a sign) where pure $\zeta$-function methods were used. I also get the same result for $a_{\zeta}$ when using the Wodzicki residue approach $[1,3,4]$. At the other extreme, setting $s=0$ $(r=\infty)$ gives a pure dimensional regulated answer, and indeed with all $\mu_{x}$ equal, $a_{s \epsilon}=0$ confirming (11). Thus the anomaly depends on the regulation used.

Another rigorous check of these results is to calculate the difference between derivatives of the $W$ functions. This defines a new type of multiplicative anomaly which I will call the " $b$-anomaly". In the mixed $s \epsilon$-regularization this is

$$
\begin{aligned}
\frac{1}{4} b_{s \epsilon}\left(\Delta_{1}^{-1}, \Delta_{2}^{-1}\right) & =\left[\frac{\partial W_{12}}{\partial\left(\delta m^{2}\right)}\right]_{s \epsilon}-\left[\frac{\partial W_{1}}{\partial\left(\delta m^{2}\right)}\right]_{s \epsilon}+\left[\frac{\partial W_{2}}{\partial\left(\delta m^{2}\right)}\right]_{s \epsilon} \\
4\left[\frac{\partial W_{j}}{\partial\left(\delta m^{2}\right)}\right]_{s \epsilon} & =\mathcal{V}_{D} \mu_{j}^{2\left(s_{j}+\epsilon_{j}\right)} \int \frac{d^{d_{j}} k}{(2 \pi)^{d_{j}}} \frac{1}{\left(k^{2}+m_{j}^{2}\right)^{1+s_{j}}}=\left\langle\phi_{j}^{2}\right\rangle \\
4\left[\frac{\partial W_{12}}{\partial\left(\delta m^{2}\right)}\right]_{s \epsilon} & =\mathcal{V}_{D} \mu_{12}^{2\left(s_{12}+\epsilon_{12}\right)} \int \frac{d^{d_{12}} k}{(2 \pi)^{d_{12}}} \frac{\left(m_{2}^{2}-m_{1}^{2}\right)}{\left(k^{4}+\left(m_{1}^{2}+m_{2}^{2}\right) k^{2}+m_{1}^{2} m_{2}^{2}\right)^{1+s_{12} / 2}}=\left\langle\left(\phi_{1}^{2}-\phi_{2}^{2}\right)\right\rangle
\end{aligned}
$$

where $\delta m^{2}=\left(m_{1}^{2}-m_{2}^{2}\right)$. In this case one can do the small $\epsilon$ and $s$ calculation with arbitrary $m_{j}^{2}$. From [18] I find that

$$
\begin{aligned}
\int_{0}^{\infty} & d x \frac{x^{-\nu-1}}{\left(1+2 \beta x+x^{2}\right)^{1 / 2+\mu}} \\
& =\frac{\left(\beta^{2}-1\right)^{\nu / 2} \Gamma(1+\mu) \Gamma(\nu+2 \mu+1) \Gamma(-\nu)}{\pi^{1 / 2} \Gamma(1 / 2+\mu) \Gamma(1+2 \mu)} \int_{-1}^{+1} d t \frac{\left(1-t^{2}\right)^{\mu-1 / 2}}{\left(t+\beta\left(\beta^{2}-1\right)^{-1 / 2}\right)^{-\nu}}
\end{aligned}
$$

Expansion of the $t$ integral in the relevant small parameters gives further integrals which can be calculated from [18]. Using these I find that

$$
\begin{aligned}
& b_{s \epsilon}\left(\Delta_{1}^{-1}, \Delta_{2}^{-1}\right)= \\
& \quad \mathcal{V}_{D} \frac{\left(m_{1}^{2}-m_{2}^{2}\right)}{C(4)}\left[\frac{1}{1+r}(\Psi(2)-\Psi(1))-\ln \left(\frac{\mu_{12}^{2}}{\mu_{1} \mu_{2}}\right)+\frac{\left(m_{1}^{2}+m_{2}^{2}\right)}{\left(m_{1}^{2}-m_{2}^{2}\right)} \ln \left(\frac{\mu_{1}}{\mu_{2}}\right)\right]
\end{aligned}
$$


where again $\epsilon=\epsilon_{x}, s=s_{x}, \forall x \in\{1,2,12\}$ is needed to ensure cancellation of the UV divergences. If I use a single renomalisation scale for the parts coming from the factored calculation (3) i.e. $\mu_{1}=\mu_{2}$, then the result is a pure function of the mass difference. In this limit $(19)$ is then identical to $2 \partial a_{s \epsilon} / \partial\left(\delta m^{2}\right)$ as expected.

So far I have only looked at the results where all the scales $\mu_{x}$ were set equal to a single independent scale parameter. However, I can exploit the fact that my results for the anomalies are for arbitrary scales, $\mu_{x}$. Suppose I choose $\mu_{12}=\mu_{1} \exp \{-(\Psi(2)-$ $\Psi(1)) /(1+r)\}$, so that in expressions like (16) we can write

$$
\mu_{12}^{2 \epsilon_{12}+2 s_{12}}=\mu_{1}^{2 \epsilon_{12}} \cdot\left(\mu_{1} e^{-(\Psi(2)-\Psi(1))}\right)^{2 s_{12}} .
$$

This unusual choice for the renormalization scales removes the anomaly for all hybrid $s \epsilon$-regularizations as (18) shows. Note that the shift is made purely in the $s$ sector indicating it is the $s$ - or $\zeta$-regularization which is causing the problem. The fact that these anomalies can be removed with a shift in renormalization scales, and hence a change in counter terms in the renormalized theory, is a clear signal that the anomaly does not contain any novel physics

One can throw further light the nature of these multiplicative anomalies by doing the reverse. Consider the results (18) and (19) for pure-dimensional regularisation $(r=$ $\infty)$. With a single scale $\mu$, the anomalies are zero. However, suppose I define a massdependent dimensional regularisation scheme where $\mu^{2}$ for a given loop integral is set equal to the average of the mass squared parameters in the integrands. It is clear from the $\mu$ dependence of (18) and (19) that the anomalies are now non-zero except when $m_{1}=m_{2}$, just as is found before with the pure $s$ - and $\zeta$-function regularisation methods. Thus it appears that multiplicative anomalies can always be interpreted as merely reflecting use of different effective renormalization scales.

One might ask if the same quantities are being compared in the different schemes. The fact that the $s \epsilon$-regularization scheme smoothly interpolates between $\zeta$-function and dimensional regularization results shows that I have a consistent definition of $[W]_{R}$ and $a_{R}$. The differences obtained in different regularization schemes are merely the expected variation found when considering unphysical objects. It is well known that such shifts can be absorbed by a redefinition of counter terms and this can be seen in the ability to remove the anomalies by changing the renormalization scales $\mu$.

These results can be summarized by a set of three necessary criteria for multiplicative anomalies to be present. Firstly, the unregulated terms of the anomaly must contain infinities. Secondly, a mass-dependent regularisation scheme must be used, and thirdly, different terms in the anomaly must contain different masses. Presumably, anomalies appear whatever physical parameter, not just mass, is involved.

If the analysis above is correct, it suggests that there are many other such anomalies associated with mass-dependent regularisation schemes. As a final check consider a mass shift $\delta m^{2}=m_{1}^{2}-m_{2}^{2}$ to a free scalar field of mass $m_{2}^{2}$. I therefore define a new " $\alpha$-anomaly" to be

$$
[\alpha(A, B)]_{R}=2\left[W_{A}\right]_{R}-2 \sum_{n=0}^{\infty}\left[\left.\frac{b^{n}}{n !} \frac{\partial^{n}}{\partial b^{n}} W_{B+b}\right|_{b=A-B}\right]_{R}
$$


where $\exp \left\{-W_{A}\right\}=\int D \phi \exp \{-\phi A \phi\}$. For the simple mass shift I set $A=\Delta_{1}^{-1}$ and $B=\Delta_{2}^{-1}$ so that $b=\delta m^{2}$. Standard integrals (17) [18] give for $D=4$ in the $s \epsilon-$ regularization scheme

$$
\alpha_{s \epsilon}\left(\Delta_{1}, \Delta_{2}\right)=-\mathcal{V}_{4} \frac{\left(m_{1}^{2}-m_{2}^{2}\right)^{2}}{C(4)}\left(\frac{1}{1+r}+\frac{m_{1}^{4}}{\left(m_{1}^{2}-m_{2}^{2}\right)^{2}} \ln \left(\frac{\mu_{1}^{2}}{\mu_{2}^{2}}\right)\right)
$$

where $r=\lim _{s, \epsilon} \epsilon / s$ and I use the same $s$ and $\epsilon$ parameters in all integrals to get the divergences to cancel. This shows exactly the same behaviour as the $a$ - and $b$-anomalies. In particular with all the renormalization scales set equal, the anomaly is zero only for $r=\infty$, pure dimensional regularization.

The $\mu$ shift discussion shows that the anomalies have no novel physical content, pace [2]. The fact that many simple schemes have no anomalies suggests a more powerful argument in support of this conclusion. It is a fundamental axiom of QFT that the physics is independent of regularization scheme (in contrast to approximate results which may depend on the scheme [9] and [19]). Since I have shown that anomalies are zero in some regularizations it follows that there can be no novel physics in non-zero anomalies in other schemes. Multiplicative anomalies are merely an annoying technical difficulty present in certain regularisation schemes. The difference in the results for anomalies would then have to be absorbed by differences in unphysical renormalization scales when ensuring physical results are invariant. This is done regularly when comparing calculations against real data [9]. Here I have explicitly demonstrated how anomalies can be removed by a suitable redefinition of renormalization scales, supporting this picture.

An important conclusion of this work is that that many different multiplicative anomalies plague calculations using $\zeta$-function and Schwinger proper time regularizations. In these schemes the physical masses are entangled with the regularisation scale since they regularize using factors such as $\left[\left(k^{2}+m^{2}\right) / \mu^{2}\right]^{-s}$ rather than $\left[k^{2} / \mu^{2}\right]^{-\epsilon}$ of dimensional regularisation. This then ensures that the regulated integrands fail to obey many algebraic identities naively satisfied by their unregulated counterparts $-\ln (A B)=\ln (A)+\ln (B)$ (the $a$ anomaly), $(A B)^{-1}=(1 / A-1 / B)(1 /(B-A))\left(b\right.$ anomaly), $\ln (1-A)^{-1}=$ $-\sum_{n} A^{n} / n$ ( $\alpha$ anomaly). The key point is that while a shift in renormalization scales $\mu$ can absorb the anomalies in such schemes, the required shift seems to depend on the expectation value being considered. So while (20) removes the $a-$ and $b$-anomalies, nothing like that removes the $\alpha$ anomaly. On the other hand, regularizations which alter the integration measure independent of physical parameters, e.g. sharp momentum cutoffs, dimensional regularization, space-time lattices, leave the integrands unchanged if a single renormalization scale $\mu$ is used, and then these never show an anomaly. Thus there seems no good reason why one should choose $\zeta$-function regularization with its multiplicative anomalies for ordinary QFT problems.

Still, all regularization schemes have some drawbacks, e.g. dimensional regularization and curved space-time, momentum cutoffs and gauge symmetry. The choice of regularization scheme is a matter of personal taste and mathematical convenience given the problem at hand. In problems involving curved space-time, $\zeta$-function methods have proved to be most useful. The crucial message is not that the multiplicative anomaly represents novel physics but rather that when $\zeta$-function methods are chosen the multiplicative anomaly must be taken into account in such calculations to get the correct physical results. In 
this sense it is of vital importance in many physical problems and requires further study.

I would like to thank A.Filippi, and R.Rivers for useful discussions, and G.Vitiello and Salerno University for stimulating some of them. This work is the result of a network supported by the European Science Foundation, with additional support from the European Commission through their Socrates and Human Capital and Mobility (CHRX-CT94-0423) programmes.

\section{References}

[1] C.Kassel, Asterisque 177 (1989) 199.

[2] E.Elizalde, L.Vanzo and S.Zerbini, Comm.Math.Phys. 194 (1998) 613 (hep-th/9701060).

[3] M.Wodzicki, Invent.Math. 75 (1984) 143.

[4] A.Connes. Non-commutative geometry (Academic Press, New York, 1994).

[5] M. Blasone and G. Vitiello, Ann.Phys. (N.Y.) 244 (1995) 283 (hep-ph/9501263).

[6] E. Alfinito, M. Blasone, A. Iorio, and G. Vitiello, Phys.Lett. B362 (1995) 91 (hep-ph/9510213).

[7] J.S.Dowker, hep-ph/9803200.

[8] G.Leibbrandt, Rev.Mod.Phys. 47 (1975) 849.

[9] Particle Data Group, Eur. Phys. J. C3 (1998) 1.

[10] J.S.Dowker and R.Critchley, Phys.Rev. D13 (1976) 3224.

[11] R.D.Ball, Phys.Rep. 182 (1989) 1.

[12] E.Elizalde, S.D.Odintsoz, A.Romeo, A.A.Bytsenko and S.Zerbini, Zeta Regularization Techniques with Applications (World Scientific, Singapore, 1994 (ISBN 9810214413)).

[13] M.Kontsevich and S.Vishik, in Functional Analysis on the Eve of the 21st Century, edited by S.Gindikin, J.Lepowsky and R.L.Wilson (Birkhäuser, Boston, 1995) (ISBN 0817637559 x v.1) Vol. 1, pp. 173.

[14] M.Wodzicki, Non-commutative Residue Chapter I, in Lecture Notes in Mathematics, ed. Yu.I. Manin, Vol. 1289, 320 (Springer-Verlag, Berlin, 1987).

[15] A.Connes and J.Lott, Nucl.Phys.Proc.Suppl. 18B 29 (1991).

[16] P.Strocchi, Elements of Quantum Mechanics of Infinite Systems (World Scientific, Singapore, 1985). 
[17] D.J.Toms, The Effective Action at Finite Temperature and Density With Application to Bose-Einstein Condensation, Report No. cond-mat/9612003.

[18] I.S.Gradshteyn, I.M. Ryzhik, Table of integrals, series and products (Academic Press, Boston, fifth edition 1994).

[19] P.M.Stevenson, Phys.Rev 12 (1981) 2916.

[20] E. Alfinito, M. Blasone, A. Iorio, and G. Vitiello, Acta Phys.Polon. B27, 1493 (1996) (hep-ph/9601354).

[21] M. Blasone, P. A. Henning, and G. Vitiello, Mixing Transformations in QFT and Neutrino Oscillations, to appear in Proceedings of Results and Perspectives in Particle Physics, La Thuile, Aosta Valley, March 1996 (hep-ph/9605335).

[22] C.Nash, Relativistic quantum fields (Academic Press, London, 1978) (ISBN 012 514350 8).

[23] E.Elizalde, J.Phys.A 27 L299 (1994) hep-th/9308028.

[24] M.Veltman Diagrammatica (C.U.P., Cambridge, 1994) (ISBN 0-521-45692-4).

[25] R.Delbourgo, Rep.Prog.Phys. 39345 (1976).

[26] M.Abramowitz and I.A. Stegun, Handbook of Mathematical Functions (Dover Publications, New York, 1965, ninth printing)

[27] E.Elizalde, A.Filippi, L.Vanzo and S.Zerbini, Phys.Rev. D57 7430 (1998) hep-th/9710171).

[28] T.S. Evans, in "Fourth Workshop on Thermal Field Theories and their Applications", 5-10 August 1995, Dalian, China, ed. Y.X.Gui, F.C.Khanna, and Z.B.Su (World Scientific, Singapore, 1996) p.283-295 (hep-ph/9510298). 


\section{A Evaluating the determinants}

Dowker [7] has made some important remarks regarding the manipulation of determinants relevant to multiplicative anomalies. Let me restate his point, extending his notation a little. Suppose the space in which we are working can be split into two parts so that each index of a matrix/operator is given by a pair $\alpha i$. For quantum field theory the $\alpha$ represents the $\mathrm{N}$-different fields and $i$ represents the space-time index running over $n^{d}$ values if we imagine working on a d-dimensional space-time lattice with $\mathrm{n}$ lattice points in each direction. Using capital letters to denote matrices/operators in this large space we have, taking $N=2$ for simplicity,

$$
M=\left(\begin{array}{ll}
a & b \\
c & d
\end{array}\right)
$$

where I will use small letters to denote matrices in $n^{d}$-dimensional space of space-time. The point made by Dowker is that

$$
D E T(M) \neq \operatorname{det}(a) \cdot \operatorname{det}(d)-\operatorname{det}(b) \cdot \operatorname{det}(c)
$$

where DET is defined as the determinant in the full $\alpha i\left(N n^{d}\right)$-dimensional space, while det denotes a determinant taken only in the $i, j$ space-time indices. As Dowker points out, with $N=2$ and $n^{d}=2$, the general expression for these matrices is a sum of 24 distinct terms on the left-hand side, while the right-hand side is a sum of only 8 distinct terms confirming the inequality given.

The focus of my letter is the case of two free scalar fields and I will first discuss this problem. Later, I will turn to the case of chemical potential which is discussed in another Elizalde et al paper [27] and which is also mentioned by Dowker. In the case of $\mathrm{N}$ free scalar fields, the propagator for $N$ fields can be represented by the matrix $\Delta_{\alpha i, \beta j}$. As the fields are free, the propagator is block diagonal in the field indices, that is for $N=2$ it is of the form:-

$$
\Delta=\left(\begin{array}{ll}
a & 0 \\
0 & d
\end{array}\right) .
$$

The $a=-\partial^{2}+m_{1}^{2}$ and $d=-\partial^{2}+m_{2}^{2}$ are $n^{d}$-dimensional matrices carrying only the space-time indices $i, j$. Now it is true in this special case that Dowker's general formula reduces to:-

$$
\operatorname{DET}(\Delta)=\operatorname{det}(a) \cdot \operatorname{det}(d) .
$$

so that in this special case equality in fact holds in (A.2). An explicit check with $N=2$, $n^{d}=2$ confirms this analysis (e.g. both sides are a sum of four terms) but it also follows from the following general analysis. Let

$$
A=\left(\begin{array}{ll}
a & 0 \\
0 & 1
\end{array}\right), \quad D=\left(\begin{array}{ll}
1 & 0 \\
0 & d
\end{array}\right)
$$

Then

$$
\begin{aligned}
\operatorname{DET}(\Delta) & =\operatorname{DET}(A \cdot D)=\operatorname{DET}(A) \cdot \operatorname{DET}(D) \\
& =[\operatorname{det}(a) \cdot \operatorname{det}(1)] \cdot[\operatorname{det}(1) \cdot \operatorname{det}(d)]=\operatorname{det}(a) \cdot \operatorname{det}(d) \cdot[\operatorname{det}(1)]^{2}
\end{aligned}
$$


and for finite dimensional matrices I have reproduced (A.4). The key point is that while Dowker makes a valid point for general matrices (A.2), it is simplifies to (A.4) for block diagonal matrices. In this case the simpler expression (A.4) is true. This is the situation encountered with free fields and this is why I used (A.4) in going from (5) to (7).

Incidently, the derivation (A.6) again illustrates why renormalisation scales are the the key to the problem. In (A.6) I have carefully shown all the factors of $\operatorname{det}(1)$ which are present. These factors are in some sense $1^{\infty}$ in quantum field theory and therefore a potential source for infinite constants. Also what do I mean by 1? Presumably this is one only with reference to some standard scale. My point is that it is precisely such scales which are difficult to understand in the zeta-function regularisation scheme and which lead to the anomaly.

In the the case of chemical potentials, studied in [27] and mentioned by Dowker [7], the propagator can be written with non-zero entries in the off-diagonal blocks, $b, c \neq 0$ in the terminology of (A.1). So it appears that Dowker's point might be of relevance. However, one can easily make such a propagator block diagonal by working in the charge eigenstate field basis (see [28]) and then the proof given here again shows once again that Dowker's point (A.2) throws no light on the multiplicative anomaly. 